\title{
Spin Hall Conductivity of a Two-Dimensional Electron Gas with Random Rashba Field
}

\author{
A. DYRDAE ${ }^{a, *}$ AND M. RATAJ ${ }^{a, b}$ \\ ${ }^{a}$ Faculty of Physics, Adam Mickiewicz University, Umultowska 85, 61-614 Poznań \\ ${ }^{c}$ The Nano-Bio-Medical Centre, Umultowska 85, 61-614 Poznań, Poland
}

\begin{abstract}
Spin Hall effect in a two-dimensional electron gas with uniform and random components of the Rashba spinorbit interaction is considered theoretically. Relaxation time due to scattering on Rashba fluctuations is also calculated. It is shown that the presence of a uniform component of Rashba coupling not only modifies relaxation time, but also suppresses the contribution to the spin Hall conductivity due to random Rashba field.
\end{abstract}

DOI: 10.12693/APhysPolA.127.499

PACS: 73.43.-f, 72.25.Hg, 73.61.Wp

\section{Introduction}

Electric field or temperature gradient can generate various spin-related phenomena in systems with spin-orbit interaction. One of the most interesting phenomena is the spin Hall effect (SHE) [1-3], where an external electric field generates spin current flowing perpendicularly to the field. Consequently, SHE gives a unique possibility to create pure spin current in nonmagnetic systems, which in turn can be used for electric manipulation with orientation of magnetic moments.

In general, Rashba spin-orbit coupling acquires not only a uniform component, but also a spatially fluctuating term (due to random distribution of dopant ions, some imperfections of quantum well interfaces etc.) that contributes to spin and momentum relaxation times, and also substantially affects spin transport. It has been shown recently that even in the system where Rashba spin-orbit interaction vanishes on average, the spatial fluctuations of the Rashba field can generate a nonzero SHE [4, 5]. In this paper we consider a more realistic case of a two-dimensional electron gas (2DEG) with both spatially uniform and random components of the Rashba field. We also determine the relaxation time due to the random Rashba term.

\section{Model}

Hamiltonian of a two-dimensional electron gas with Rashba spin-orbit interaction takes the form (we use the units with $\hbar=1$ )

$$
\begin{aligned}
H & =\sum_{\boldsymbol{k} \boldsymbol{k}^{\prime}} \Psi_{\boldsymbol{k}}^{\dagger}\left(\frac{k^{2}}{2 m} \delta_{\boldsymbol{k} \boldsymbol{k}^{\prime}}+\alpha\left(\sigma_{x} k_{y}-\sigma_{y} k_{x}\right) \delta_{\boldsymbol{k} \boldsymbol{k}^{\prime}}\right. \\
& \left.+\frac{\lambda_{\boldsymbol{k} \boldsymbol{k}^{\prime}}}{2}\left[\sigma_{x}\left(k_{y}+k_{y}^{\prime}\right)-\sigma_{y}\left(k_{x}+k_{x}^{\prime}\right)\right]\right) \Psi_{\boldsymbol{k}^{\prime}},
\end{aligned}
$$

where the first term (diagonal in the spin space) stands for the kinetic part with $m$ being the electron effective

\footnotetext{
* corresponding author; e-mail: adyrdal@amu.edu.pl
}

mass, the second term describes the uniform (constant) part of the Rashba spin-orbit interaction with the Rashba parameter $\alpha$, while the last term represents the spatial fluctuations of the Rashba field, which will be treated perturbatively. The parameter $\lambda_{\boldsymbol{k} \boldsymbol{k}^{\prime}}$ describes the random Rashba spin-orbit interaction. Spatial average of this parameter vanishes, $\langle\lambda(\boldsymbol{r})\rangle=0$. However, the correlation function, $\left\langle\lambda(\boldsymbol{r}) \lambda\left(\boldsymbol{r}^{\prime}\right)\right\rangle=C\left(\boldsymbol{r}-\boldsymbol{r}^{\prime}\right)$, is nonzero. Fourier transform $C_{q}$ of $C\left(\boldsymbol{r}-\boldsymbol{r}^{\prime}\right)$ takes the form [6]

$$
C_{q}=\left|\lambda_{q}\right|^{2}=2 \pi\left\langle\lambda^{2}\right\rangle(2 R)^{2} \mathrm{e}^{-2 q R},
$$

where $R$ is the spatial scale of the fluctuations, and $\boldsymbol{q}=\boldsymbol{k}-\boldsymbol{k}^{\prime}$ is the momentum change due to scattering by fluctuations of the Rashba field.

The Green functions, $G_{\boldsymbol{k}}^{R / A}(\varepsilon)$, corresponding to the nonperturbative part of the Hamiltonian (1), is a matrix in the spin space and can be written as

$$
G_{\boldsymbol{k}}^{R / A}=G_{\boldsymbol{k} 0}^{R / A} \sigma_{0}+G_{\boldsymbol{k} x}^{R / A} \sigma_{x}+G_{\boldsymbol{k} y}^{R / A} \sigma_{y},
$$

with

$$
\begin{aligned}
G_{\boldsymbol{k} 0}^{R / A} & =\frac{1}{2}\left(G_{\boldsymbol{k}+}^{R / A}+G_{\boldsymbol{k}-}^{R / A}\right), \\
G_{\boldsymbol{k} x, y}^{R / A} & = \pm \frac{k_{y, x}}{2 k}\left(G_{\boldsymbol{k}+}^{R / A}-G_{\boldsymbol{k}-}^{R / A}\right),
\end{aligned}
$$

where $G_{k \pm}^{R / A}=\left(\varepsilon-E_{k \pm} \pm \mathrm{i} \Gamma\right)^{-1}, E_{k \pm}=k^{2} / 2 m \pm \alpha k$ are the two electron bands of $2 \mathrm{DEG}$ with constant Rashba spin-orbit interaction, $k_{i}(i=x, y)$ is the $i$-th component of the vector $\boldsymbol{k}, \sigma_{n}$ with $n=(0, x, y, z)$ are the unit and Pauli matrices in the spin space, and $\Gamma=1 / 2 \tau$ with $\tau$ being the total relaxation time. Both $\Gamma$ and $\tau$ depend on $k$, which is not indicated explicitly.

\section{Relaxation time}

The total relaxation time includes scattering from impurities, $\tau_{i}$, and scattering from the random Rashba field, $\tau_{r}: \tau^{-1}=\tau_{i}^{-1}+\tau_{r}^{-1}$. To determine $\tau_{r}$ we calculate the self energy in the Born approximation:

$$
\Sigma_{\boldsymbol{k}}^{R}=\int \frac{\mathrm{d}^{2} \boldsymbol{k}^{\prime}}{(2 \pi)^{2}} V_{\boldsymbol{k} \boldsymbol{k}^{\prime}} G_{\boldsymbol{k}^{\prime}}^{R} V_{\boldsymbol{k}^{\prime} \boldsymbol{k}} .
$$

Here $V_{\boldsymbol{k} \boldsymbol{k}^{\prime}}$ is the perturbation due to random Rashba field, the last term in Eq. (1). Taking into account the explicit form of $V_{\boldsymbol{k} \boldsymbol{k}^{\prime}}$ and Green function $G_{\boldsymbol{k}^{\prime}}^{R}$, one finds 


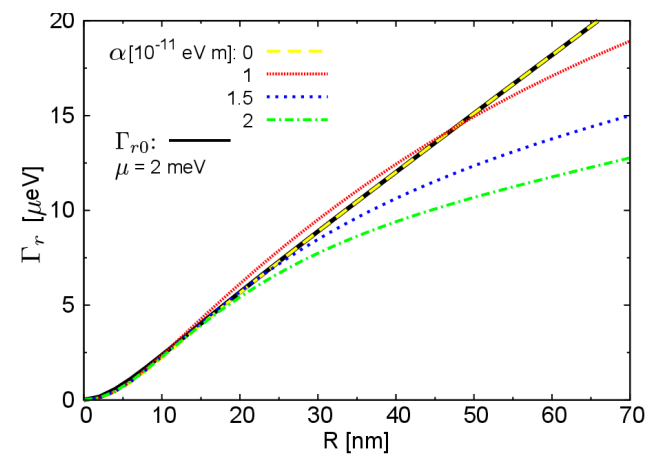

Fig. 1. Relaxation rate (for subband $E_{\boldsymbol{k}+}$ ) due to random Rashba field plotted as a function of the correlation length $R$ for indicated values of $\mu$ and $\alpha$. The other parameters are $\sqrt{\left\langle\lambda^{2}\right\rangle}=1.5 \times 10^{-12} \mathrm{eVm}$ and $m=0.05 m_{0}$.

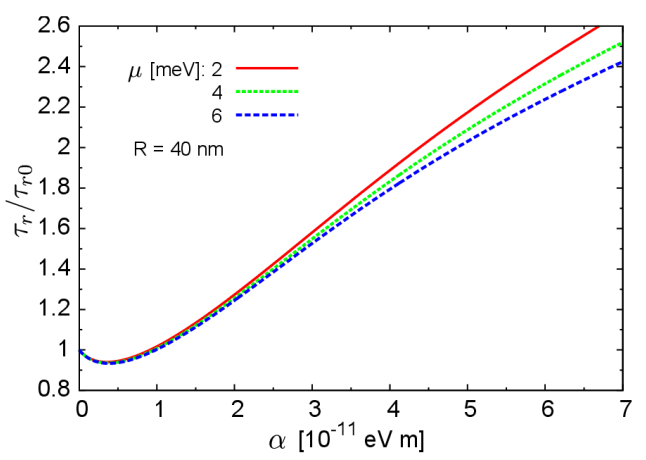

Fig. 2. Relaxation time (for subband $E_{k+}$ ) due to random Rashba field plotted as a function of parameter $\alpha$ for indicated values of $\mu$ and $R$. The other parameters are as in Fig. 1.

that $\Sigma_{\boldsymbol{k}}^{R}=\Sigma_{0 k}^{R} \sigma_{0}$. The imaginary part of $\Sigma_{0 k}^{R}$ determines the relaxation rate: $\operatorname{Im} \Sigma_{0 k}^{R}=\Gamma_{r}$ and $\Gamma_{r}=1 / 2 \tau_{r}$. The explicit form of $\Gamma_{r}$ at the Fermi level, $\mu$, is given as

$$
\begin{aligned}
& \Gamma_{r}(\mu)=\frac{1}{4 \pi} \int \mathrm{d} q q \int \mathrm{d} \theta \frac{C_{q}}{8}\left(4 k_{\mathrm{F}}^{2}+q^{2}-4 k_{\mathrm{F}} q \cos \theta\right) \\
& \quad \times\left[\delta\left(\mu-E_{\boldsymbol{k}-\boldsymbol{q}+}\right)+\delta\left(\mu-E_{\boldsymbol{k}-\boldsymbol{q}-}\right)\right],
\end{aligned}
$$

where $\theta$ is the angle between vectors $\boldsymbol{k}$ and $\boldsymbol{q}$. Note, the vector $\boldsymbol{k}$ is on the Fermi circle of one of the subbands with the corresponding Fermi wavevectors equal to $\sqrt{m^{2} \alpha^{2}+2 m \mu} \mp m \alpha$. In the limit of $\alpha \rightarrow 0$, the relaxation rate $\Gamma_{r}$ reduces to $\Gamma_{r 0}$ given by the expression [7]

$$
\Gamma_{r 0}=\frac{m}{2 \pi} \int_{0}^{2 k_{\mathrm{F}}} C_{q} \sqrt{4 k_{\mathrm{F}}^{2}-q^{2}} \mathrm{~d} q .
$$

The relaxation rate is plotted in Fig. 1 as a function of the correlation length $R$ for indicated values of the uniform Rashba coupling parameter $\alpha$. One can see that for long-range correlations $\Gamma_{r}$ increases linearly with $R$. When the system is in a dirty limit, $R \rightarrow 0$, the relaxation rate tends to zero as $R^{2}$. The relaxation time due to fluctuations of the Rashba field decreases with increasing uniform Rashba coupling parameter $\alpha$. This behavior is shown explicitly in Fig. 2, where the relaxation time is plotted as a function of the parameter $\alpha$. This figure shows that for $\alpha>10^{-11} \mathrm{eVm}$, the relaxation time due to random Rashba field is longer in a system with both uniform and random components of spin-orbit field than in a system with random component only.

\section{Spin Hall effect}

According to the Kubo-Streda formula [8], we write the spin Hall conductivity as a sum of two components: $\sigma_{x y}^{s_{z}}=\sigma_{x y}^{s_{z} \mathrm{I}}+\sigma_{x y}^{s_{z} \mathrm{II}}$. The component $\sigma_{x y}^{s_{z} \mathrm{II}}$ is the contribution to the spin Hall conductivity, which depends on states below the Fermi level. This contribution vanishes in the case considered. Thus,

$$
\sigma_{x y}^{s_{z}}=\sigma_{x y}^{s_{z} \mathrm{I}}=\frac{e}{2 \pi} \int \frac{\mathrm{d}^{2} \boldsymbol{k}}{(2 \pi)^{2}} \operatorname{Tr}\left\{\hat{j}_{x}^{s_{z}} G^{R}(\mu) \hat{v}_{y} G^{A}(\mu)\right\} .
$$

The spin current operator is $\hat{j}_{i}^{s_{n}}=\left[\hat{j}_{i}, \hat{s}_{n}\right]_{+} / 2 e$, where $\hat{s}_{n}=\sigma_{n} / 2$ is the n-th component of the electron spin and $\hat{j}_{i}=e \hat{v}_{i}$ is the charge current operator with $\hat{v}_{i}=\partial H / \partial k_{i}$ being the electron velocity operator. The perturbation expansion for the Green functions in the above expression leads to a series of Feynman diagrams depicted in Fig. 3, that contribute to the spin Hall conductivity.

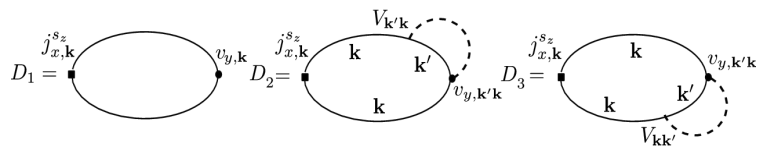

Fig. 3. The Feynman diagrams contributing to the spin Hall conductivity in the dc limit.

Therefore, the spin Hall conductivity can be written as: $\sigma_{x y}^{s z}=\frac{e}{2 \pi} \operatorname{Tr} \sum_{\boldsymbol{k}_{\boldsymbol{k}^{\prime}}}\left(D_{1}+D_{2}+D_{3}\right)$. The diagram $D_{1}$ is the so-called bare bubble diagram and represents the universal intrinsic contribution to the spin Hall conductivity [9]: $\sigma_{x y}^{s_{z} \text { int }}=e / 8 \pi$. The diagrams $D_{2}$ and $D_{3}$ are associated with the presence of anomalous velocity vertex and they are a consequence of the random Rashba field.

The spin Hall conductivity can be thus written as $\sigma_{x y}^{s_{z}}=\frac{e}{8 \pi}+\delta \sigma_{x y}^{s z}$, where $\delta \sigma_{x y}^{s z}=\frac{e}{2 \pi} \operatorname{Tr} \sum_{k k^{\prime}}\left(D_{2}+D_{3}\right)$. Writing explicitly contributions from the diagrams $D_{2}$ and $D_{3}$ and taking into account that $\boldsymbol{k}^{\prime}=\boldsymbol{k}-\boldsymbol{q}$, we get the following expression:

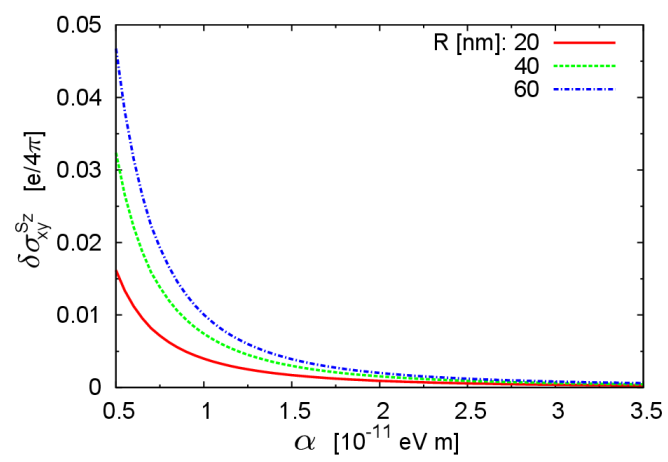

Fig. 4. The contribution $\delta \sigma_{x y}^{s z}$ as a function of uniform Rashba coupling parameter $\alpha$ for indicated values of $R$ and $\mu=4 \mathrm{meV}$. The relaxation rate $\Gamma=0.5 \mathrm{meV}$. The other parameters are as in Fig. 1. 


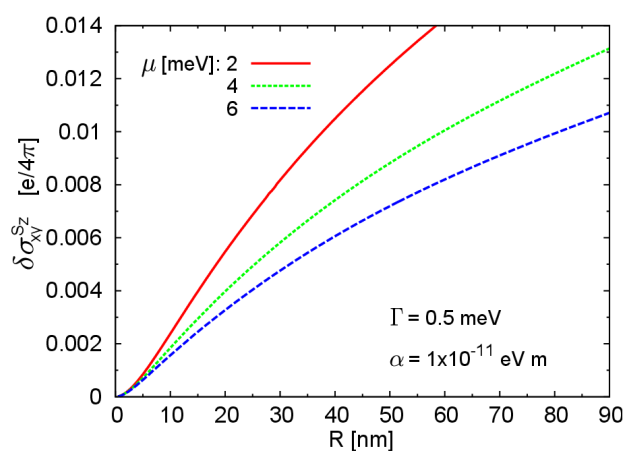

Fig. 5. The contribution $\delta \sigma_{x y}^{s_{z}}$ as a function of the correlation parameter $R$ for indicated values of $\mu$ and $\alpha$. The relaxation rate $\Gamma=0.5 \mathrm{meV}$. The other parameters are as in Fig. 1.

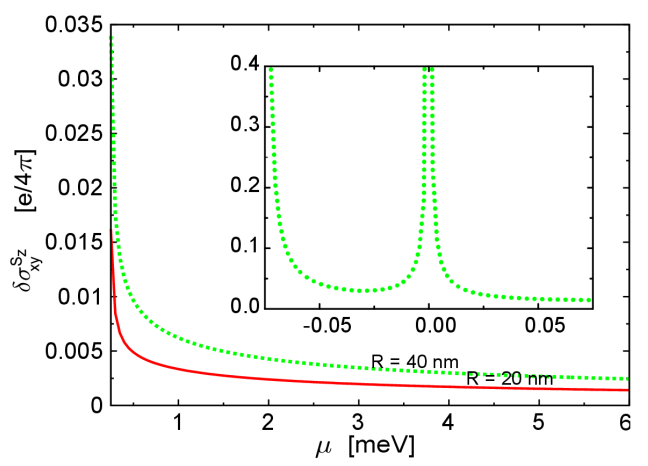

Fig. 6. The contribution $\delta \sigma_{x y}^{s z}$ plotted as a function of the chemical potential. The relaxation rate $\Gamma=$ $0.5 \mathrm{meV}$. The other parameters are as in Fig. 1.

$$
\begin{aligned}
& \delta \sigma^{s_{z}} x y=\frac{e}{2 \pi} \int \frac{\mathrm{d} k k}{(2 \pi)^{2}} \int \frac{\mathrm{d} q q}{(2 \pi)^{2}} \int \mathrm{d} \theta \int \mathrm{d} \phi \frac{C_{q}}{4 m} \\
& \times\left[k_{x}\left(2 k_{y}-q_{y}\right)\left(t_{1} \cos \phi-f_{y} t_{2}\right)-k_{x}\left(2 k_{x}-q_{x}\right)\right. \\
& \left.\times\left(t_{3}\left(f_{x} \sin \phi-f_{y} \cos \phi\right)-t_{4}\right)\right],
\end{aligned}
$$

where

$$
\begin{aligned}
& t_{1,3}=-\mathrm{i} \frac{\pi}{\Gamma}\left(G_{\boldsymbol{k}_{+}}^{A} G_{\boldsymbol{k}-}^{R}-G_{\boldsymbol{k}_{-}}^{A} G_{\boldsymbol{k}+}^{R}\right) \\
& \quad \times\left[\left(\mu-E_{\boldsymbol{k}-\boldsymbol{q}+}\right) \delta\left(\mu-E_{\boldsymbol{k}-\boldsymbol{q}+}\right)\right. \\
& \left.\quad \pm\left(\mu-E_{\boldsymbol{k}-\boldsymbol{q}-}\right) \delta\left(\mu-E_{\boldsymbol{k}-\boldsymbol{q}-}\right)\right] \\
& t_{2,4}=\pi\left(G_{\boldsymbol{k}+}^{A} G_{\boldsymbol{k}-}^{R}+G_{\boldsymbol{k}-}^{A} G_{\boldsymbol{k}+}^{R}\right) \\
& \quad \times\left[\delta\left(\mu-E_{\boldsymbol{k}-\boldsymbol{q}+}\right) \mp \delta\left(\mu-E_{\boldsymbol{k}-\boldsymbol{q}-}\right)\right]
\end{aligned}
$$

and

$$
\begin{aligned}
f_{x} & =\frac{(k-q \cos \theta) \sin \phi-q \cos \phi \sin \theta}{\sqrt{k^{2}-2 k q \cos \theta+q^{2}}} \\
f_{y} & =\frac{(k-q \cos \theta) \cos \phi-q \sin \phi \sin \theta}{\sqrt{k^{2}-2 k q \cos \theta+q^{2}}} .
\end{aligned}
$$

The corresponding numerical results obtained for $\delta \sigma_{x y}^{s_{z}}$ are presented in Figs. 4-6. Here, we assume that $\tau_{r}^{-1} \rightarrow 0$ and the main contribution to $\Gamma$, treated here as a constant parameter, comes from scattering by impurities.
In Fig. 4 the contribution to the spin Hall effect due to random component of the Rashba field is plotted as a function of the uniform Rashba coupling parameter $\alpha$. The contribution $\delta \sigma_{x y}^{s z}$ tends to zero for sufficiently large values of the parameter $\alpha$. Behavior of $\delta \sigma_{x y}^{s_{z}}$ as a function of the correlation length $R$ is shown in Fig. 5. In the limit of long-range correlations, the spin Hall conductivity is a linear function of $R$, but in the dirty limit $\delta \sigma_{x y}^{s_{z}}$ decreases with $R$ as $R^{2}$. These results are consistent with the results obtained for $2 \mathrm{DEG}$ in the presence of random Rashba field only [4] and for 2DEG with Dresselhaus spin-orbit interaction and random Rashba field [10]. In Fig. 5, in turn, the contribution $\delta \sigma_{x y}^{s_{z}}$ is depicted as a function of the Fermi level. The spin Hall conductivity decreases with increasing chemical potential. The sharp peak appears at the minimum of the lower subband. For $\mu=0$ the spin Hall conductivity reveals a singularity.

\section{Summary}

We have analyzed SHE in a two-dimensional electron gas with spatially uniform and random components of the Rashba spin-orbit field. The spin Hall conductivity has an universal intrinsic contribution equal to $e / 8 \pi$, and an additional term, $\delta \sigma_{x y}^{s_{z}}$. The contribution $\delta \sigma_{x y}^{s_{z}}$ strongly depends on the spatial length of correlations and is suppressed for sufficiently large value of the Rashba coupling constant $\alpha$.

\section{Acknowledgments}

The authors would like to thank J. Barnaś for discussion. This work has been supported by National Center of Science (NCN, Poland) as a research project No. DEC$2011 / 03 /$ N/ST3/02353 for years 2012-2014 and by the EU under ESF - Operational Programme Human Capital - POKL.04.01.01-00-133/09-00.

\section{References}

[1] M.I. Dyakonov, V.I. Perel, Pis'ma Z. Eksp. Teor. Fiz. 13, 657 (1971); JETP Lett. 13, 467 (1971).

[2] J.E. Hirsch, Phys. Rev. Lett. 83, 1834 (1999).

[3] G. Vignale, J. Supercond. Nov. Magn. 23, 3 (2010).

[4] V.K. Dugaev, M. Inglot, E.Ya. Sherman, J. Barnaś, Phys. Rev. B 82, 121310(R) (2010).

[5] A. Dyrdał, J. Barnaś, Phys. Rev. B 86, 161401(R) (2012).

[6] M.M. Glazov, E.Ya. Sherman, V.K. Dugaev, Physica E 42, 2157 (2010).

[7] V.K. Dugaev, E.Ya. Sherman, V.I. Ivanov, J. Barnaś, Phys. Rev. B 80, 081301(R) (2009).

[8] P. Streda, J. Phys. C: Solid State Phys. 15, L717 (1982).

[9] J. Sinova, D. Culcer, Q. Niu, N.A. Synitsyn, T. Jungwirth, A.H. MacDonald, Phys. Rev. Lett. 92126603 (2004).

[10] A. Dyrdał, J. Barnaś, Acta Phys. Pol. A 1221016 (2012). 